\title{
Modeling Sea Level During El Niño
}

\author{
MARK A. CANE' \\ Department of Meteorology and Physical Oceanography, Massachusetts Institute of Technology. Cambridge, MA 02139
}

(Manuscript received 27 August 1983, in final form 28 September 1984)

\begin{abstract}
We test the hypothesis that sea level variations associated with El Niño events are a response to wind changes in the central Pacific and that the signal is transmitted to the coast of South America by packets of equatorial Kelvin waves. A linear model is forced by wind stress anomalies composited from six El Niños occurring between 1951 and 1972 (Rasmusson and Carpenter). Model sea level is compared with similar composites of tidegage measurements from selected equatorial Pacific stations.

Although several vertical modes are included in the calculation, only the two gravest baroclinic modes make significant contributions to the sea level signal. Model results duplicate the pattern and timing of the observed sea level anomalies but amplitudes are systematically low. This is especially true at central and western Pacific stations, and it is suggested that the poor quality of the forcing data may be at fault there. Results at the east provide better support for the theory: in particular, the twin-peaked signal characteristic of El Niño sea-level anomalies is reproduced. The second peak is shown to be a response to the massive collapse of the trades occurring in the middle of the El Niño year and its amplitude is correctly hindcast by the model. The first peak is a response to the weaker wind changes occurring in the boreal fall preceding $\mathrm{El}$ Niño; its calculated amplitude is too small. The implication of this discrepancy is that the linear Kelvin wave theory will have to be modified if it is to account for the initial El Niño warming.
\end{abstract}

\section{Introduction}

Originally El Niño was the name that fishermen of Peru and Equador gave to the southward flowing current of warm water that appears off their coast every year shortly after Christmas. Later the name was taken to apply to the catastrophic warming events associated with mass mortality of birds and fish. It is now widely appreciated that the coastal warmings are part of a global syndrome of anomalous conditions in both the atmosphere and oceans. A thorough theory for El Niño events will require an account of the feedbacks between the atmosphere and ocean that comprise and probably drive the El Niño-Southern Oscillation cycle.

We are far from having such a theory at present. There are some mechanistic scenarios for the whole cycle, but none that is specific enough to be considered a testable hypothesis. However, there are reasonably precise theories for certain aspects of the cycle, and the intent of the work reported here is to provide a test of one of these. This theory takes the wind changes as given and purports to explain the sea level and sea surface temperature (SST) changes that they induce, especially at the South American coast. The notation that the oceanographic events are attributable

\footnotetext{
'Present affiliation: Lamont-Doherty Geological Observatory, Physical Oceanography, Palisades, NY 10964.
}

to changes in the tropical wind stress (as opposed to, for example, surface heat flux or purely oceanographic causes) appears to originate with Bjerknes (1966), but was first developed to a specific theory by Wyrtki (1975); also see Godfrey (1975), McCreary (1976), Hurlburt et al. (1976). This theory identifies the relevant wind changes as the ones along the equator in the remote central and western Pacific. Wind changes along the South American coast were found to be negligible, ruling out variations in locally induced coastal upwelling as the source of the El Niño signal. The remote theory goes further in identifying the messenger carrying the signal from the mid-Pacific to the South American coast as the equatorial Kelvin wave. (More precisely, packets of equatorial Kelvin waves.) The implication is that anomalous advection by mean currents and other nonlinear effects, while certainly present (cf. Wyrtki's, 1977, discussion of the North Equatorial Countercurrent), are not important influences on the coastal response.

In this work we calculate the oceanic response to observed winds on the basis of linear theory. The wind stress driving is derived from the composite $\mathrm{El}$ Niño surface wind anomaly fields constructed by Rasmusson and Carpenter (1982). We focus the comparisons with oceanographic measurements on sea level changes. A variety of oceanographic data describing past El Niños is available, but only sea level and SST provide sufficient coverage to permit 
meaningful comparisons. Sea surface temperature is influenced by a number of factors in addition to dynamics, notably surface heat flux and nonadiabatic mixed layer physics (i.e., the degree of surface layer mixing). Quantitative values of surface heat flux anomalies are notoriously uncertain (e.g., Leetmaa, 1983 ) and the parameterization of mixed layer processes can hardly be said to be well established, especially in the presence of the unusually strong upwelling velocities and vertical shears found in equatorial flows. Sea surface temperature data alone does not provide a clearcut test of the dynamical hypothesis described above. Sea level variations, on the other hand, are little affected by nonadiabatic processes and should be accounted for by a purely dynamical theory. Furthermore, numerical experiments with nonlinear models (Cane, 1979; Philander, 1979) strongly suggest that variations in such essentially integral quantities as sea level and dynamic topography are well predicted by linear dynamics. We conclude that sea level provides a good first test of the hypothesis sketched above. Linear dynamical theory should be expected to explain sea level anomalies: if it fails there are fewer extenuating arguments in defense of the theory than is the case with other variables.

There have been only two previous calculations of El Niño response using observed winds. Gill (1983) combined observations of winds with coastal sea level records from the $1972 \mathrm{El}$ Niño to predict other variables (e.g., surface currents, SST). More relevant here is the series of calculations carried out at Florida State University (FSU) and reported in Busalacchi and O'Brien (1981) and Busalacchi et al. (1983). A linear shallow water model with parameters matched to mid-Pacific values for the first baroclinic mode was driven by two decades (1961-80) of monthly surface wind stress fields. (The analysis procedure used to obtain these fields is described in Goldenberg and O'Brien, 1981.) These landmark calculations were the first to show a causal relation between wind changes and oceanic response during El Niño. By correlating model pycnocline anomaly with sea level observations it was concluded that the model accounted for $25 \%$ of the observed variance. The model clearly underpredicts the amplitude of the sea level signal. According to linear theory sea level is significantly influenced by more modes than the single one simulated in the FSU model; so some of the unexplained variance may be due to the extreme simplicity of the model. The present calculation eliminates that source of error by calculating the response as a sum of many modes. Another major source of error lies in the fact that no analysis procedure can entirely overcome the sparse coverage of the surface wind data set. In the present study we use composites constructed from six El Niño events, a procedure that reduces the error in the wind field by paying a heavy cost in temporal coverage. (For further discussion see Rasmusson and Carpenter, 1982.) Our results then show substantial successes in accounting for $\mathrm{El}$ Niño sea level changes, but also reveal systematic discrepancies which we feel cannot properly be attributed to shortcomings in the data. These are discussed further in the concluding section.

The plan of the remainder of the paper is as follows. The next section briefly discusses the data used while the following one presents the necessary theory and briefly describes the model used to perform the calculations. Section 4 describes the results and Section 5 considers their implications.

\section{Data}

Two kinds of data are needed in this study: wind stress fields and sea level measurements. Our wind fields derive from the composites constructed by Rasmusson and Carpenter (1982) and described in their paper. Briefly, for each box in a $2^{\circ} \times 2^{\circ}$ latitude-longitude grid the monthly mean over the entire period of the data record (1947-72) is found. They then determine anomalies relative to this mean for each three year period centered on the year of the six major El Niños occurring in this period (1951, $1953,1957,1965,1969,1972$ ). Since coverage for any given year is scanty, they then composite the data by combining data for the same month from all six events. (A detailed justification for this procedure is given in their paper.) Even so, coverage remains inadequate, so each month's data point is finally computed to be the average of the data in the three month period centered on the nominal month. Thus a total of 18 months worth of data go into each datum of the composite.

The origin of the sea level data is the tide gage network maintained by Dr. Klaus Wyrtki (cf. Wyrtki, 1979). The stations we have chosen to study are those shown in Fig. 1; all of the equatorial Pacific stations with reasonably long time series are included. A few of the time series are quite long, but we consider only data taken from the same period as the wind data, 1947-72. Composite El Niño sea-level anomalies were computed in a manner similar to the wind composites, with the following differences. First, the data set we obtained contained the mean for each month, rather than the individual observations throughout the month. Second, only the data for the nominal month went into the composite as compared with the three consecutive months of the wind composite. Thus each data point of the sea level composite was constructed from the monthly means from each of the six El Niños. [There are often fewer than six points because years are missing.]

The results of this compositing are shown in Fig. 1. First, consider the five stations along the South American coast (Tumaco, La Libertad, Talara, Chim- 

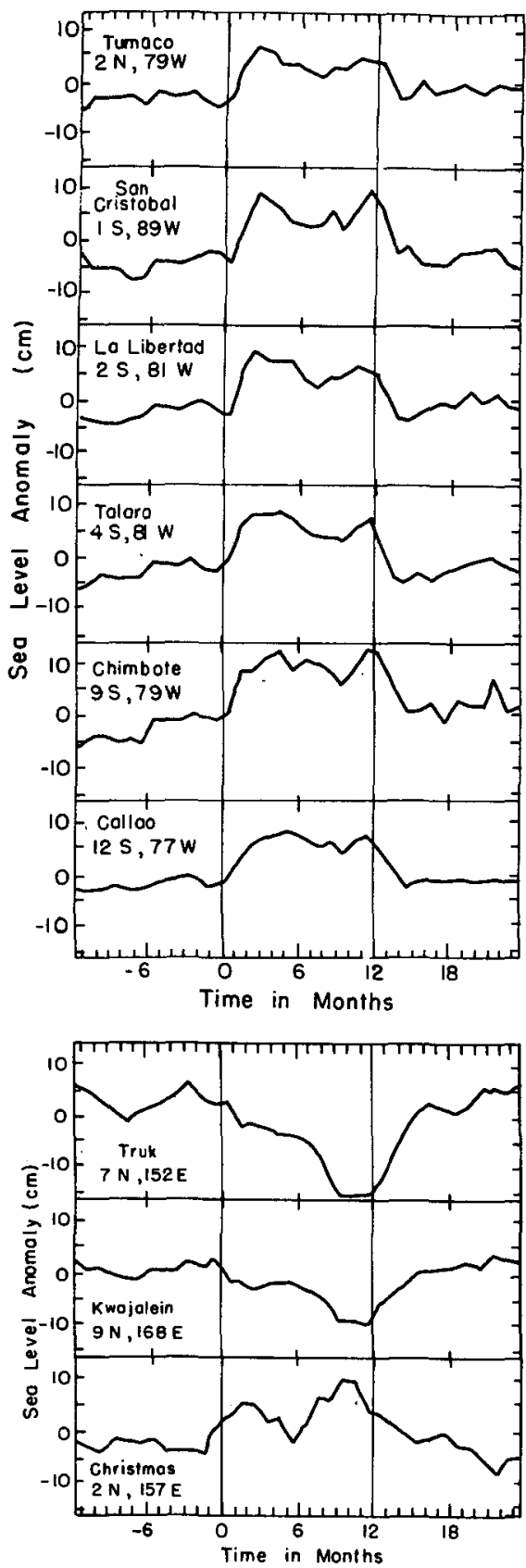

FIG. 1. Observed composite sea-level anomalies (cm) at selected equatorial Pacific stations the year before El Niño, the year of El Niño, and the year following El Niño.

bote, Callao). The pattern and amplitude are similar at all of these, the distinguishing features being two peaks during the El Niño year. There tends to be a sharp rise from the beginning of the year until about March, with the peak persisting for several months. The second peak occurs at the end of the year and continues into the following year. The two peaks are close in amplitude, with the first peak being a bit higher. The monthly data used shows no obvious phase differences as one proceeds poleward down the coast. The record at San Cristobal in the Galapagos Islands about $1000 \mathrm{~km}$ from the coast, is similar in pattern, phase and amplitude.

Now consider the stations to the west beginning with Christmas Island, the one farthest to the east and closest to the equator. A twin-peaked pattern is again apparent, but the pattern is phase shifted one to two months earlier. The second peak is comparable in magnitude to those at the coast but the first peak is substantially smaller. The stations considered thus far clearly suggest a signal moving eastward along the equator to the coast. The off-equatorial stations further to the west (Truk and Kwajalein) show a very different pattern. Sea level falls throughout the El Niño year, with the rate increasing sharply in the second half of the year (cf. Meyers, 1982).

The general sense of all the stations is an increase in mass in the eastern equatorial Pacific and a decrease in the west (Wyrtki, 1977, 1979). The obvious inference is that this comes about through an eastward transfer of mass; from Wyrtki (1979) one can estimate that during the $1976 \mathrm{El}$ Niño this transfer amounted to an average of $27 \times 10^{6} \mathrm{~m}^{3} \mathrm{~s}^{-1}$ through the year. A principal contention of the theory under consideration is that such a transfer does take place and that it is effected by packets of equatorial Kelvin waves. The plausibility of this idea is enhanced by the fact that equatorial Kelvin waves have been observed to cross the Pacific from the dateline to the Galapagos (see Knox and Halpern, 1982, or Eriksen et al., 1983). In the next section we develop the theory needed to calculate the Kelvin wave amplitude in response to the wind.

\section{Theory}

We will review the general linear theory rather succinctly, lingering over only those less familiar aspects essential to what follows. [An excellent account of the standard linear theory may be found in Moore and Philander, 1977; a recent example of an application appears in Gent et al., 1983.]

\section{a. Vertical decomposition}

Variables describing the circulation may be written as a sum of vertical standing modes; for the horizontal velocity components and the pressure,

$$
\begin{aligned}
& {\left[u(x, y, z, t), v(x, y, z, t), \frac{1}{\rho} P(x, y, z, t)\right]} \\
& \quad=\sum_{m=0}^{\infty}\left[u_{m}^{\prime}(x, y, t), v_{m}^{\prime}(x, y, t), g h_{m}^{\prime}(x, y, t)\right] A_{m}(z) .
\end{aligned}
$$

The vertical structure functions $\left[A_{m}(z)\right]$ are the solutions to an eigenvalue-eigenfunction problem that depends on the stratification through the horizontally 
averaged buoyancy frequency $N(z)$ and on the top and bottom boundary conditions. The associated eigenvalues $H_{m}$ are referred to as equivalent depths. The set $\left(A_{m}\right)$ is orthogonal so the representation (1) is unique. For each $m\left(u_{m}^{\prime}, v_{m}^{\prime}, h_{m}^{\prime}\right)$ satisfy the shallow water equations for an ocean of depth $H_{m}$. Suppose the ocean circulation is driven by a stress divergence $\partial \tau(x, y, z, t) / \partial z$; then in view of (1) the $m$ th system of shallow water equations is forced by a body force of magnitude

$$
\tau_{m}(x, y, t)=\frac{1}{D} \int_{-D}^{0} \frac{\partial \tau}{\partial z} A_{m}(z) d z,
$$

where $D$ is the depth of the ocean and $A_{m}(z)$ has been normalized so that

$$
\int_{-D}^{0} A_{m}^{2}(z) d z=D .
$$

We now make the common assumptions that

(i) There is a surface mixed layer of depth $d$ which is so well mixed that $N^{2}(z)=0$ for $0>z>-d$;

(ii) The stress $\tau$ vanishes below the mixed layer.

As a consequence of (i) $A_{m}(z)$ is constant in the mixed layer; i.e. $A_{m}(z)=A_{m}(0)$ for $z>-d$. Integrating (2) by parts and then making use of this fact together with (ii) yields

$$
\tau_{m}(x, y, t)=\tau(x, y, 0, t) \frac{A_{m}(0)}{D} .
$$

Finally we equate the stress at $z=0$ to the surface wind stress. The result (4) would also be obtained by adopting Lighthill's (1969) assumption that the wind stress acts as a body force over the mixed layer and not below.

We now choose a scale $\tilde{\tau}$ for the wind stress and define scales $U_{m}, D_{m}$ and nondimensional variables $u_{m}, v_{m}, h_{m}$ by the relations

where

$$
\left(u_{m}^{\prime}, v_{m}^{\prime}\right)=U_{m}\left(u_{m}, v_{m}\right), h_{m}^{\prime}=D_{m} h_{m},
$$

$$
D_{m}=A_{m}(0) L_{m}\left[\frac{\tilde{\tau} / \rho}{g D}\right] \text {, }
$$

$$
U_{m}=g D_{m} / c_{m}=c_{m} D_{m} / H_{m},
$$

and $L_{m}$ and $c_{m}$ are the standard equatorial length scale and wave speed (see Table 1). By scaling distance by $L_{m}$ and time by $T_{m}$ we obtain the same formal equations for each $m$ :

$$
\left.\begin{array}{l}
\frac{\partial}{\partial t} u_{m}-y v_{m}+\frac{\partial}{\partial x} h_{m}=\tau^{(x)}(x, y, t) \\
\frac{\partial}{\partial t} v_{m}+y u_{m}+\frac{\partial}{\partial y} h_{m}=\tau^{(y)}(x, y, t) \\
\frac{\partial}{\partial t} h_{m}+\frac{\partial}{\partial x} u_{m}+\frac{\partial}{\partial y} v_{m}=0
\end{array}\right\}
$$

No stratification dependent parameters appear explicitly in (6) but since the length and time scales depend on the internal wave speed $c_{m}$ the wind stress pattern and the basin size are different for each $m$. Hence the response will be different for each mode.

Since the model ocean is hydrostatic the sea level $\eta$ is directly related to the surface pressure; using (1) and (5):

$$
\eta=\frac{1}{\rho g} P(z=0)=\sum_{m=0}^{\infty} h_{m}^{\prime} A_{m}(0)=\sum_{m=0}^{\infty} D_{m} A_{m}(0) h_{m} .
$$

Defining a sea level scale $\eta_{m}$ by

$$
\begin{aligned}
\eta_{m} & \equiv D_{m} A_{m}(0)=\left[\frac{\tilde{\tau} / \rho}{g D}\right] A_{m}{ }^{2}(0) L_{m}, \\
\eta & =\sum_{m=0}^{\infty} \eta_{m} h_{m} .
\end{aligned}
$$

Obviously, the contribution each mode makes to the

\begin{tabular}{|c|c|c|c|c|c|c|c|c|c|}
\hline & \multicolumn{9}{|c|}{$m$} \\
\hline & $\mathbf{0}$ & 1 & 2 & 3 & 4 & 5 & 6 & 7 & 8 \\
\hline Equivalent depth $H_{m}(\mathrm{~cm})$ & $5 \times 10^{5}$ & 86 & 32 & 13 & 7.0 & 4.3 & 3.3 & 2.3 & 1.8 \\
\hline Surface amplitude $A_{m}(0)$ & 1.00 & 4.22 & 4.02 & 2.05 & 1.61 & 2.08 & 2.10 & 1.50 & 1.22 \\
\hline Wave speed, $c_{m}=\left(g H_{m}\right)^{1 / 2}(\mathrm{~m} / \mathrm{s})$ & 222. & 2.91 & 1.78 & 1.13 & 0.83 & 0.65 & 0.57 & 0.47 & 0.42 \\
\hline Time scale, $T_{m}=\left(\beta c_{m}\right)^{-1 / 2}$ (day) & 0.16 & 1.42 & 1.80 & 2.28 & 2.66 & 3.00 & 3.21 & 3.53 & 3.74 \\
\hline Length scale, $L_{m}=\left(c_{m} / \beta\right)^{1 / 2}(\mathrm{~km})$ & 3117 & 357 & 279 & 222 & 190 & 168 & 158 & 144 & 135 \\
\hline Sea level scale $\eta_{m}[$ Eq. (7)] $(\mathrm{cm})$ & 0.32 & 0.65 & 0.46 & 0.10 & 0.05 & 0.07 & 0.07 & 0.03 & 0.01 \\
\hline Sea level estimate $\eta_{m}^{*}$ [Eq. (12)] (cm) & 0.03 & 1.82 & 1.65 & 0.43 & 0.26 & 0.44 & 0.45 & 0.23 & 0.15 \\
\hline
\end{tabular}
sea level signal depends strongly on the surface value of the horizontal structure function, $A_{m}(0)$. Table 1 gives values of $A_{m}(0)$ and $\eta_{m}$ for a stratification typical of the Pacific at $179^{\circ} \mathrm{W}$ near the equator. [Values are based on an average of CTD casts from $1.5^{\circ} \mathrm{N}$ to $1.5^{\circ} \mathrm{S}$ at $179^{\circ} \mathrm{W}$, as described by Eriksen et al., 1983 . $D=5000 \mathrm{~m}$ and we took $\tilde{\tau}=0.05 \mathrm{~Pa}$. It would appear that many modes might make a significant contribution to sea level. However, $\eta$ also depends

TABLE 1. Normal mode characteristics. Values for $H_{m}, A_{m}$ were based on stratification typical of the equatorial Pacific near the dateline (Eriksen et al., 1983). The depth $D=5000 \mathrm{~m} . \tau \sim 0.5 \mathrm{dyn} \mathrm{cm}^{-2}$ and $L_{F} \sim 1000 \mathrm{~km}$ were used for the sea-level parameters. 
on the $h_{m}$, which depend on $H_{m}$ (through the length and time scales) and on the structure of the wind stress $\tau$. In the next subsection we will make scaling estimates of these effects, estimates which our direct calculations will show to be reasonable. It turns out that the $m=1$ and $m=2$ modes are by far the most significant.

We now digress a bit to relate the foregoing to the usual $1 \frac{1}{2}$ layer model in which a layer of depth $D^{\prime}$ and density $\rho-\Delta \rho$ is assumed to lie over a deep motionless layer of density $\rho$. Motions in this model are governed by the shallow water system (6) if $c_{m}{ }^{2}$ $=g \Delta \rho / \rho D^{\prime}$. The value of sea level so obtained will be the same as the contribution of the $m$ th mode if $D^{\prime}=D / A_{m}^{2}(0)$. For the stratification we use, this value is $281 \mathrm{~m}$ for the first baroclinic model while $c_{1}$ $=2.91 \mathrm{~m} \mathrm{~s}^{-1}$. In the FSU calculations referred to in the Introduction, $D^{\prime}=300 \mathrm{~m}$ while $c_{m}=2.45 \mathrm{~m} \mathrm{~s}^{-1}$ (cf. Busalacchi and O'Brien, 1981). The wave speed is thus about $16 \%$ slower than our $c_{1}$ while their sea level amplitude is about $7 \%$ higher than our first mode sea level scale $\eta_{1}$. We will find that the first mode typically contributes about half of the sea level signal.

\section{b. Horizontal structure}

In order to find the horizontal structure of each mode we must consider the solution of the shallow water system (6) for each $m$. The nature of the wind stress data means that the time scale of the motions can be no less than a month. (The minimum will be more nearly two months in view of the averaging.) For the modes that influence sea level, the graver baroclinic modes, the motions will be low frequency. in the sense that their time scale is long compared with the internal time scale of the mode (cf. Table 1). An extensive theory for such motions has been developed by Cane and Sarachik (1976, 1977, 1979, 1981) and we will quote some relevant results here. At low frequencies there are three important components to the ocean's response: (i) eastward propagating long equatorial Kelvin waves, (ii) long, westward propagating Rossby waves, and (iii) short, eastward propagating Rossby waves. The wavelengths of the last of these are too short for them to be effectively excited by the wind stress forcing. They can be generated at the western boundary reflections of incident long waves, but their group velocities are too low to permit them to penetrate into the interior. In sum, they are important at the western boundary when they make up the boundary current but have no effect elsewhere (cf. Cane and Sarachik, 1981, p. 754ff).

Now consider the Kelvin wave and its relation to the response at the eastern boundary, $x=X_{E}$. The (model) height at the eastern boundary, $h_{m}\left(X_{E}, y, t\right)$, depends on the amplitude of the Kelvin waves incident there, their long Rossby wave reflections, and the sea surface setup due to the local longshore winds. The last effect is negligible for El Niño anomalies because there are no such wind changes during El Niño (Wyrtki, 1975; Enfield, 1981a; Rasmusson and Carpenter, 1982). Writing the Kelvin wave in the form

$$
\begin{aligned}
\left(u_{m}\right)_{\text {Kelvin }}=\left(h_{m}\right)_{\text {Kelvin }}=a_{m}(x, t) 2^{-1 / 2} \psi_{0}(y) \\
=a_{m}(x, t) 2^{-1 / 2} \pi^{-1 / 4} e^{-1 / 2 y^{2}},
\end{aligned}
$$

we have the asymptotic, low-frequency result (Cane and Sarachik, 1977, p. 406)

$$
h_{m}\left(X_{E}, \dot{y}, t\right)=\pi^{-1 / 4} a_{m}\left(X_{E}, t\right)=\sqrt{2}\left(h_{m}\right)_{\text {Kelvin }} .
$$

There are two significant implications of this result: 1) $h_{m}\left(X_{E}, y, t\right)$ depends only on the Kelvin wave amplitude at the coast at time $t, a_{m}\left(X_{E}, t\right)$ and 2) $h_{m}\left(X_{E}\right)$ is independent of latitude. Recall that for the coastal stations shown in Fig. 1 that sea level changes are approximately in-phase along the coast and the amplitudes are independent of latitude.

The amplitude $a_{m}$ is determined from the wave equation

$$
\left(\frac{\partial}{\partial t}+\frac{\partial}{\partial x}\right) a_{m}(x, t)=b_{m}(x, t)
$$

where

$$
b_{m}(x, t)=2^{-1 / 2} \int_{-\infty}^{+\infty} \psi_{0}(y) \tau^{(x)}(x, y, t) d y
$$

(e.g., cf. the Appendix to Gent et al., 1983, or McCreary, 1976). Here $\tau^{(x)}$ is the nondimensional zonal component of the surface wind stress. Equation (9) is easily solved; at the eastern boundary $x=X_{E}$,

$$
\begin{aligned}
& \pi^{1 / 4} h_{m}\left(X_{E}, t\right)=a_{m}\left(X_{E}, t\right)=a_{m}\left[x=X_{w},\right. \\
& \left.t-\left(X_{E}-X_{w}\right)\right]+\int_{X_{w}}^{X_{E}} b_{m}\left[x, t-\left(X_{E}-x\right)\right] d x,
\end{aligned}
$$

where $X_{w}$ is the longitude of the western boundary of the ocean. According to (11), sea level changes at the South American coast will depend on the zonal wind stress integrated over the length of the Pacific. The contribution from longitude $x$ is lagged to allow time for the Kelvin wave to travel from $x$ to $X_{E}$. For the baroclinic modes, only the winds near the equator contribute to the integral in (11) because the wind contribution is weighted by a narrow Gaussian centered on the equator [viz. (10) and note from Table 1 that $L_{m}$ is less than $400 \mathrm{~km}$ for each of the baroclinic modes].

The magnitude of the integral in (11) may be estimated as follows. Take the length scale of the forcing $\tau^{(x)}$ to be $L_{F}$; its nondimensional value for mode $m$ is then $L_{F} / L_{m}$. The $x$-integral in (11) is $\sim b_{m} L_{F} / L_{m}$, with $b_{m}$ computed from (10). As long as $L_{m}<L_{F}$, then $\tau^{(x)}(y)$ is approximately constant where the Gaussian $\psi_{0}(y)$ is $O(1)$ so $b_{m} \sim \tau^{(x)}(y=0)$. This 
will be the case for the baroclinic modes. If $L_{F} \ll L_{m}$, as for the barotropic mode, then $b_{m} \sim \tau^{(x)} L_{F} / L_{m}$. Combining these estimates, the overall contribution to sea level of the $m$ th mode, $\eta_{m}^{*}$, is expected to be

$$
\begin{aligned}
\eta_{m}^{*} & =\eta_{m} \frac{L_{F}}{L_{m}} \min \left(1, L_{F} / L_{m}\right) \\
& =A_{m}{ }^{2}(0) L_{F}\left[\frac{\tau / \rho}{g D}\right] \min \left[1, L_{F} / L_{m}\right] .
\end{aligned}
$$

Values based on a wind stress magnitude of $0.05 \mathrm{~Pa}$ $\mathrm{cm}^{-2}$ and a wind scale of $1000 \mathrm{~km}$ are given in Table 1. The barotropic mode is clearly negligible and will not be considered further. The first two baroclinic modes are obviously dominant but contributions from modes 3-7 might possibly be significant.

If $h_{m}\left(X_{E}\right)$ were completely determined by the integral in (11) it would be a trivial matter to calculate sea level at the eastern boundary from (11), (10), and (7) given the zonal wind stress over the equatorial ocean. However, the Kelvin wave amplitude at the western boundary, $a_{m}\left(X_{W}\right)$, must also be known. This is determined by the western boundary condition that the integral of the mass flux normal to the coast vanishes (Cane and Sarachik, 1977, p. 404), so (the integral of) the incident mass flux is required. The analytic expression for this in terms of the wind field involves a sum of complicated convolution integrals. (The Appendix to Gent et al., 1983, gives the solution in the case when the forcing is periodic. It suggests the complexity of the more general time-dependent solution.) Rather than use this expression we evaluate $a_{m}\left(X_{W}\right)$ by a numerical procedure described by Cane and Patton (1984). Inter alia we obtain $u_{m}, h_{m}$, etc. over the entire model basin, allowing comparisons with sea level records away from the coast and the equator. Briefly, the model uses a numerical scheme that is second-order in space and time to calculate the long Rossby wave part of the flow. The Kelvin wave component is calculated exactly by integrating along characteristics. The low-frequency, long-wave approximation is made and the scheme is implicit so the timestep is limited by accuracy rather than stability requirements. In the present calculation a 10-day time step is used, adequate to resolve the forcing data. The model domain is a rectangle from $124^{\circ} \mathrm{E}$ to $80^{\circ} \mathrm{W}$ and $20^{\circ} \mathrm{S}$ to $20^{\circ} \mathrm{N}$ with a rectangle crudely representing Mexico removed in the northeast corner. The southwest corner of this rectangle is at $95^{\circ} \mathrm{W}$, $15^{\circ} \mathrm{N}$. Variables are located on a staggered grid with a $1 \times 0.5 \mathrm{deg}$ longitude-latitude spacing between like variables. As noted in the Introduction, the model was forced by the composite wind stress anomalies of Rasmusson and Carpenter (1982). The drag coefficient was taken to have the relatively large value of $2.8 \times 10^{-3}$. The only friction in the calculation is a Rayleigh friction with a five-year spindown time.

\section{Results}

Figure 2 shows the amplitude of the first baroclinic Kelvin mode at the two ends of the model Pacific Ocean for the three years centered on the El Niño year. The dotted lines connect the point at the west with the point it influences at the east [i.e., the time separation is $C_{1}^{-1}\left(X_{E}-X_{w}\right)$; cf. (11)]. The value of the Kelvin wave amplitude at the west coast makes a relatively small contribution to its value at the east. The dropoff from March to June is the only significant exception to this statement. These statements are characteristic of the other modes we have calculated $(m=2,3,4)$.

Most of the height change at the east must be due to the zonal winds near the equator. According to (10) and (11) the measure of this influence in time and space is $b_{m}(x, t)$; contours of $b_{1}(x, t)$ are plotted in Fig. 3. (Contours of $b_{2}$ are very similar; as shown below, the higher modes make a much smaller contribution.) The curve at the right of the figure is a plot of $a_{1}\left(X_{E}, t\right)$, as in Fig. 2. The dotted lines indicate the path of a Kelvin wave through this phase plane. The rise in $a_{1}$ at the beginning of the El Niño year is primarily due to the change in the winds in the vicinity of the dateline. In the composite there are substantial easterly anomalies in this region from the summer to the fall of the year preceding El Niño. By November they have relaxed back to normal, remaining about normal east of the dateline and becoming anomalously westerly to the west of it. The latter anomaly appears to be the primary cause of the first peak in the El Niño year, though there is also a smaller contribution from anomalies occurring about

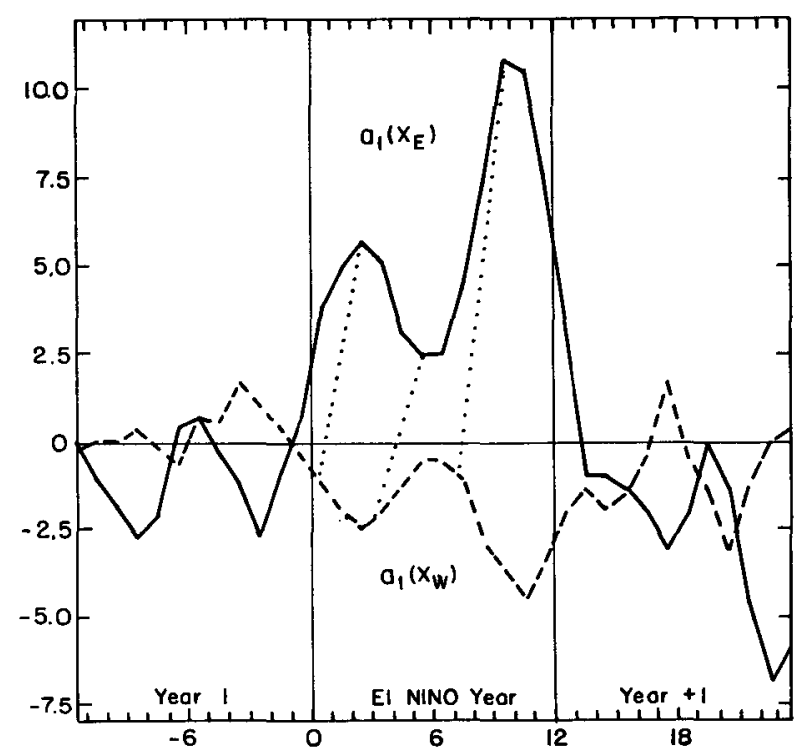

FIG. 2. Calculated first baroclinic-mode Kelvin wave amplitude at the eastern end $X_{E}$ and western end $X_{k^{\prime}}$ of the model Pacific Ocean. 


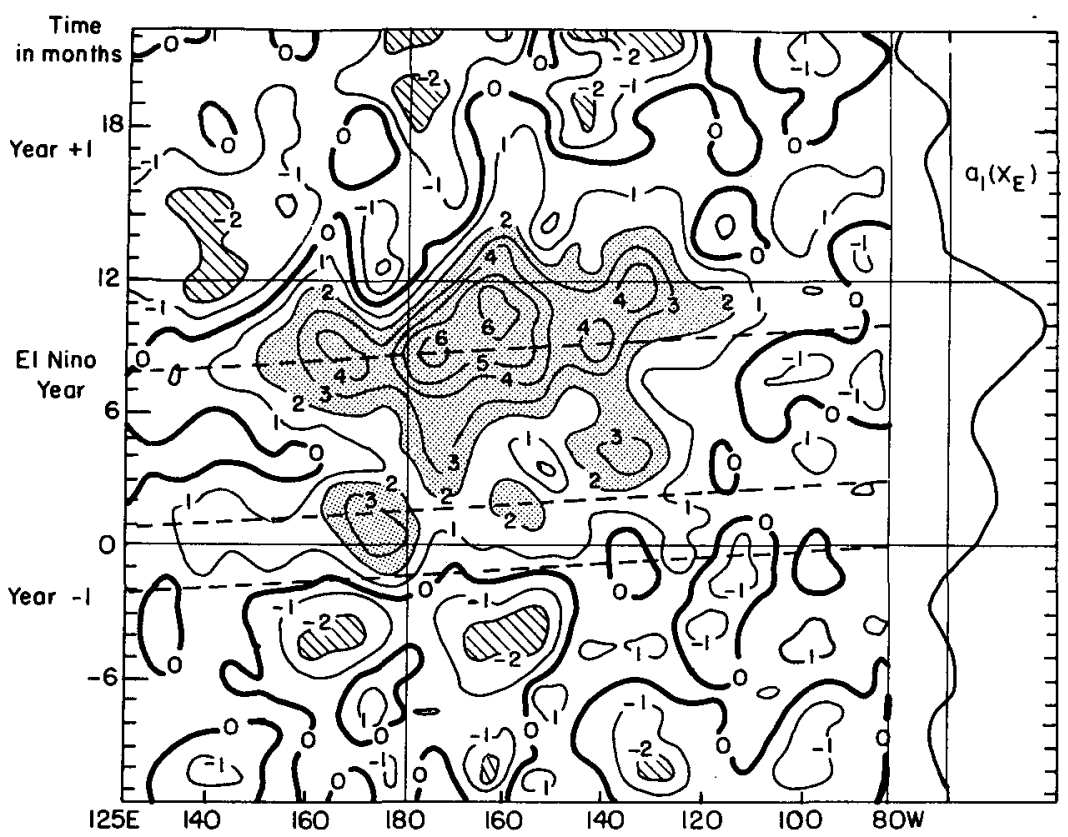

FiG. 3. The first baroclinic-mode Kelvin wave forcing $b_{1}(x, t)$ [Eq. (10)]. Dotted lines indicate the path of a Kelvin wave. The curve on the right is $a_{1}\left(X_{E}\right)$ as in Fig. 2.

February around $160^{\circ} \mathrm{W}$. The second, stronger peak centered in October of the El Niño year is clearly a result of the massive collapse of the trades in the Central Pacific.

In Fig. 4 we consider the contributions of the various modes to the total sea level signal. At each station we have plotted $\eta$, the total sea level signal, and $A_{m}(0) D_{m} h_{m}$, the contribution of the $m$ th mode to that signal, for the first four baroclinic modes. The plots bear out the a priori estimates of the last section that the first two baroclinic modes, and only the first two modes, matter to the sea level response. Those estimates understated the dominance of the first two modes, primarily because the scaling for the integral in (11) didn't fully capture the inability of the slowly traveling higher modes to make constructive use of relatively short-lived spatially coherent wind changes.

The first panel of Fig. 4 shows the results for the coastal station at $2^{\circ} \mathrm{S}$, La Libertad. Model results for the other coastal stations are indistinguishable at the resolution of the plot, verifying that longshore coastal winds make a negligible contribution to sea level changes. The second mode makes a slightly larger contribution than the first, but the magnitude of the two are not very different. The second mode lags the first by about a month, so the sea level peaks are somewhat broader than either individual contribution. The same general features found at La Libertad also characterize San Cristobal in the Galapagos Islands.

The remaining panels of Fig. 4 show stations farther to the west, where the dynamics of the sea level response is more complicated. For points on or near the equator (e.g., Christmas Island) it will be dominated by the Kelvin mode and the gravest Rossby mode. The response is influenced by signals propagating in from both east and west as well as by local forcing. For points off the equator (e.g., Truk, Kwajalein) the response is due to local forcing and to Rossby waves propagating in from the east. It is particularly sensitive to the curl of the wind stress, which cannot be determined accurately from available wind stress data. The only possible Kelvin wave influence is indirect, via the circuitous and slow route eastward along the equator to the coast and then westward via reflected Rossby waves.

The response at Truk, the furthest west of our stations, is predominantly first mode. Kwajalein resembles the eastern stations in being equally influenced by the first and second modes through the El Niño year, and in having the two contributions take a similar form. In the year following El Niño the first mode takes over. At Christmas the second mode response is much stronger than the first. In sum, there is no consistent pattern in the western ocean beyond the fact that the first two baroclinic modes are the important ones.

Figure 5 compares the model results with the composites based on tidegage measurements. There is a clear tendency for the pattern of the calculated sea level to resemble the observations; the correlation coefficient between the two would be high (cf. Busalacchi and O'Brien, 1981). The amplitude of the model sea level variations is smaller than the observed, however. The best agreement is at the South American 


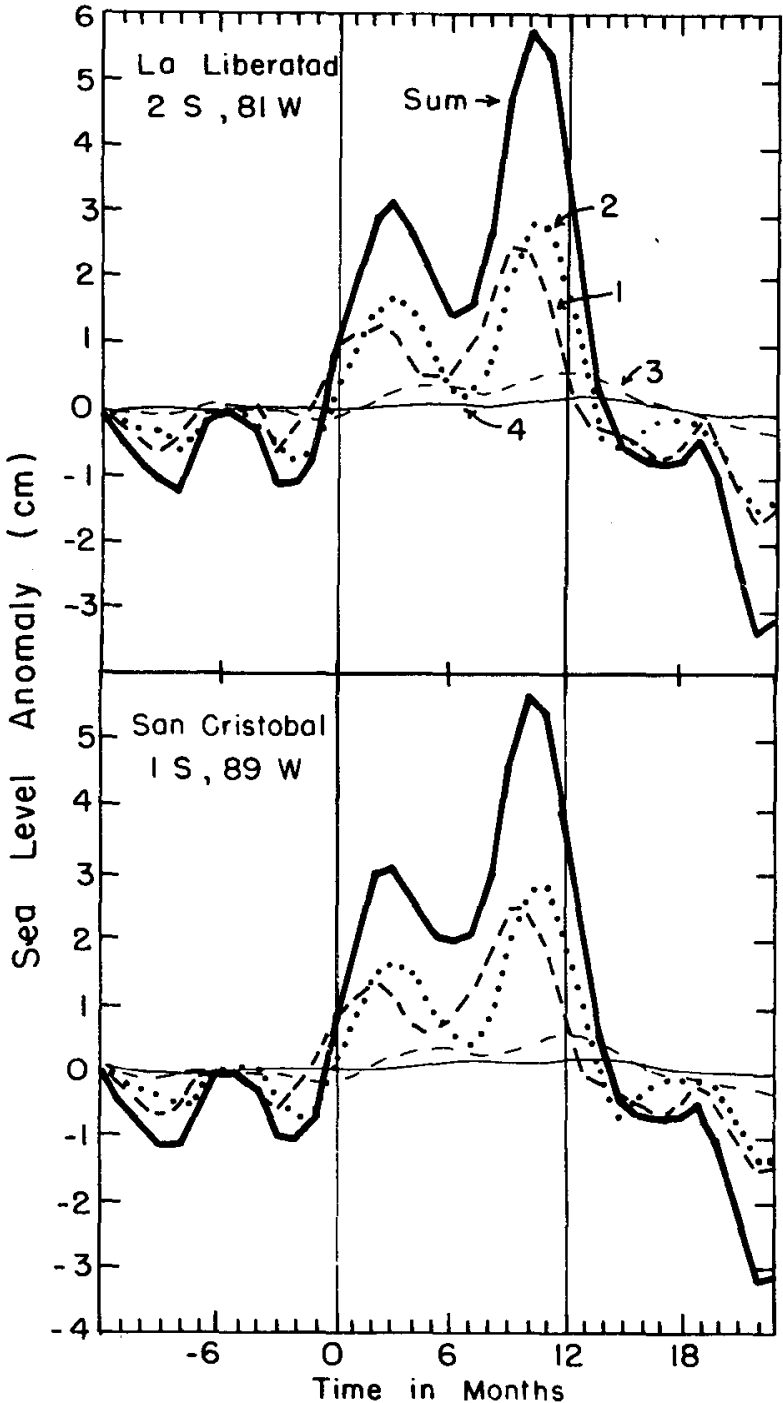

FIG. 4. Model sea-level anomalies $(\mathrm{cm})$ at selected stations. Heavy solid line is total sea level anomaly; other lines are the contribution of individual modes: Ist mode (dashed line); 2nd mode (dotted line); 3rd mode (light solid line); 4th mode (light dashed line).

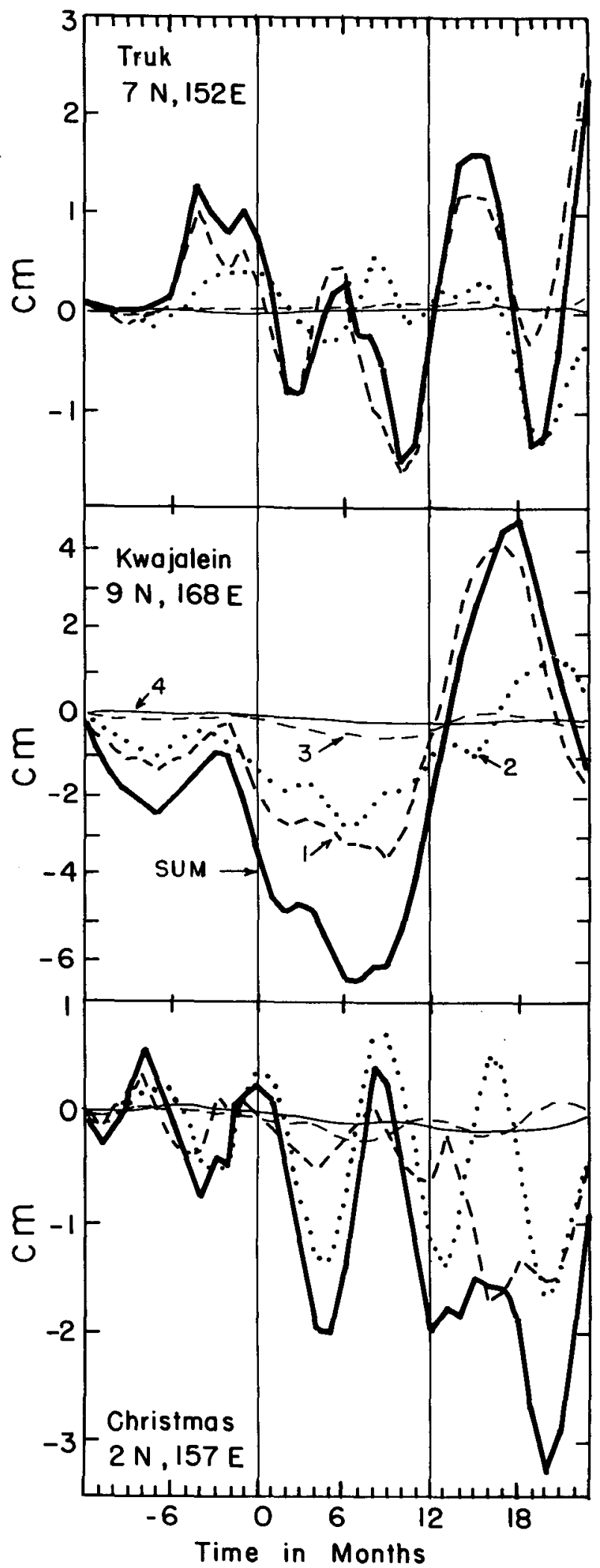




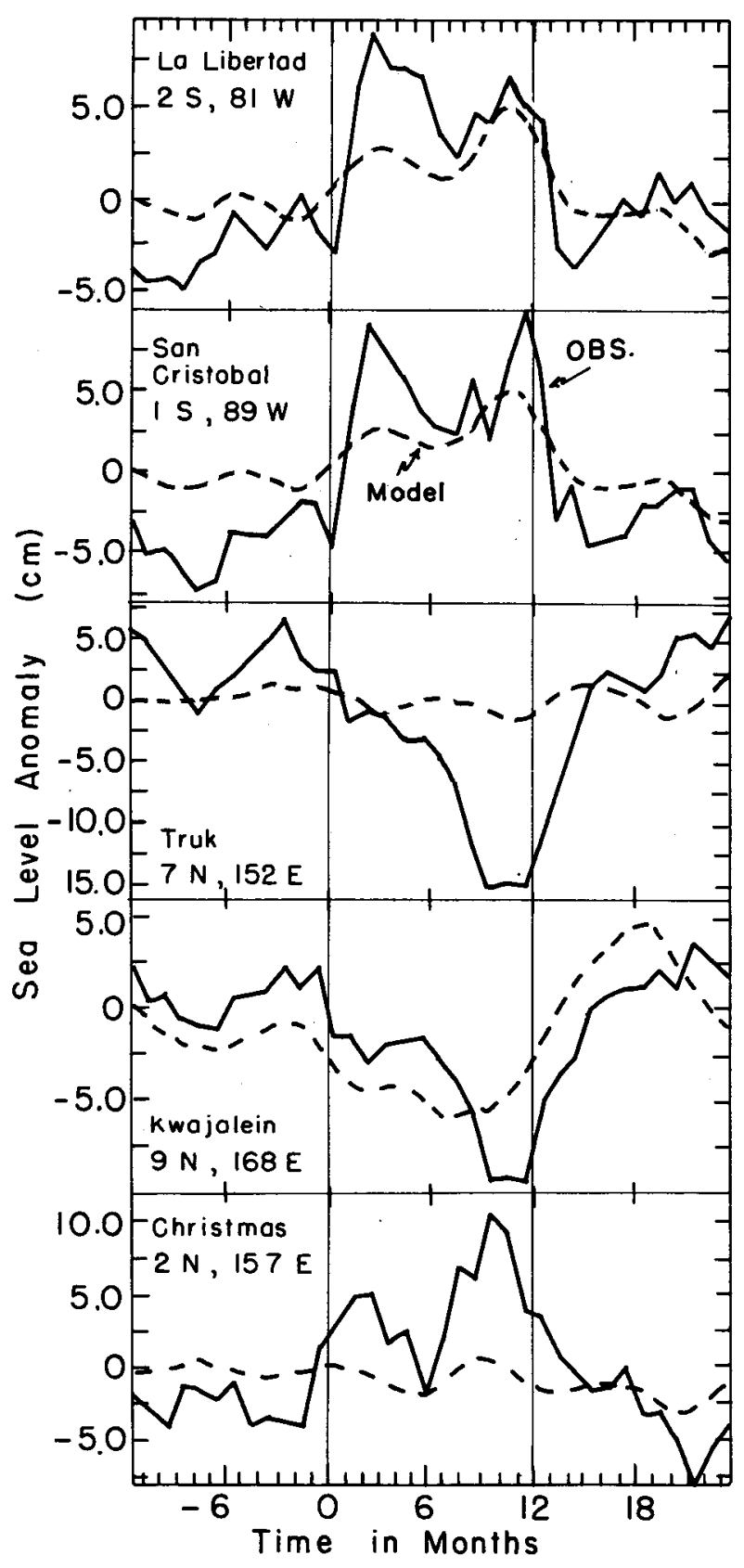

FIG. 5. Observed (solid line) and model (dashed line) sea level anomalies $(\mathrm{cm})$.

coast where, as discussed before, the physics governing sea level anomalies is simplest. At La Libertad the two peaked structure is clear in both data and model and the timing of the two is the same, though the model sea level starts to rise a bit earlier than the observed. It is striking that the amplitude of the second peak is the same in both, but that of the first peak is larger in the observations and smaller in the model. In fact, the first peak amplitude is the only serious discrepancy over the $2 \frac{1 / 2}{2}$ year period beginning in the summer preceding El Niño. [In view of the lack of wind information before January of year 1, the model cannot be expected to be correct during the first 6 months.] The story at San Cristobal in the Galapagos is much the same except that the second peak here is also underpredicted.

Results in the west are more erratic and generally worse. Truk is badly underpredicted though the pattern and timing of the model is similar to the observations (as is true in Busalacchi and O'Brien, 1981). Model results at Kwajalein are somewhat weak and tend to be about two months early, but agreement is generally good. There is no reason to suppose the physics governing the response at Truk would be different than at Kwajalein: both are out of the equatorial waveguide and are west of the major wind changes. The poor quality of the forcing data in a region where the response depends on wind stress curl seems a plausible source for the discrepancies. The response at Christmas Island, near the equator in a region of strong wind changes, is also anemic.

\section{Discussion}

The intent of the calculations reported here is to test the most widely accepted theory for the oceanic response during El Niño events: that the changes at the South American coast are caused by changes in the zonal wind stress in the central Pacific and that their influence is transmitted across the Pacific by equatorial Kelvin waves. We have used sea level as the measure of the ocean's response because, as discussed in the Introduction, it provides the clearest test of such a dynamical theory. If the theory fails to account for sea level changes it can't be invoked to explain anything else, such as SST anomalies. If it does account for sea level changes at the coast, then the coastal SST warming may be attributed to the same cause. The Kelvin waves that raise sea level suppress the thermocline along the coast (consistent with Enfield, 1981b). Thus, although the winds remain favorable for upwelling, the water advected toward the surface and entrained into the surface mixed layer is warmer than normal. In addition, the arrival of equatorial Kelvin waves results in poleward advection along the coast, bringing in warmer water from north of the Galapagos Front, which is south of the equator early in the year.

The poor quality of the data is the most obvious reason for any disagreement between the calculated sea level and observations. We believe that the Rasmusson and Carpenter (1982) composite wind anomaly data set is the best objectively analyzed wind field currently available, but it is clear that the winds are severely undersampled, especially in the crucial area west of the dateline. (See their paper for further discussion.) Figure 3 provides evidence of the problem. The contoured field of $b_{1}$ is very noisy despite the 
fact that each data point is made from 18 months of observational data which is then further averaged meridionally (in a Gaussian weighted way).

There are also shortcomings in the sea level data, but a more serious problem for our purpose is the mismatch between sea level and wind data: the temporal distributions of the two data sets are very different. The six El Niños in the composite are similar in many respects but they are not the same with regard to the location and timing of wind anomalies. The distribution of wind data is bound to weight the component El Niños differently in different locations and only by accident would any of these weightings be the same as their sea level counterparts. In addition, the anomalies used here are based on means for wind and sea level which do not correspond to one another.

In view of the shortcomings of the data our conclusions about the validity of the theory, both positive and negative, must be tentative. We judge the theory to be successful in accounting for the sea level changes at the South American coast. In particular, it reproduces the twin peaked pattern characteristic of $\mathrm{El}$ Niño. If this judgment is accepted a number of conclusions follow. The first and second baroclinic modes and only these modes are important in determining the sea level response. The coastal response is a result of equatorial Kelvin wave packets impinging on the coast. This statement is consistent with the observation that the El Niño sea-level signal is very nearly the same in phase and amplitude at all stations along the coast (Fig. 1). The Kelvin wave amplitudes (and hence the coastal signal) are determined almost entirely by the zonal winds within a few degrees of the equator. All of the essential information is presented in Fig. 3 which shows the appropriate functional of the zonal wind stress anomaly. The initial El Niño peak results from the westerly anomaly that develops in the vicinity of the dateline in the boreal fall preceding El Niño. The second peak is due to the massive collapse of the trades in the central Pacific which begins in the middle of the El Niño year.

The dynamics of the response along the South American coast are extremely simple, depending only on the Kelvin wave amplitude given by (11) and (10). The response in the western Pacific is more complex, especially off the equator where it is determined by combinations of Rossby waves. These are influenced by the wind stress curl. Thus while the eastern response depends on integrals of the wind stress, the off-equatorial response depends on derivatives of the wind stress field, making it more sensitive to errors in the data. The model results in the western Pacific are erratic, to say the least. Sea level at Kwajalein is reasonably well simulated while results at Truk are poor. Since the governing physics at the two islands ought to be similar it is difficult to see reasons for this difference beyond errors in the data.

Our results do contain discrepancies which we believe should not be attributed to data errors. Although we use a relatively high value for the drag coefficient, calculated sea level is consistently low. One source of this systematic difference is the steric effect associated with the advection of abnormally warm water into the surface mixed layer. Warming a $25 \mathrm{~m}$ layer by $3^{\circ} \mathrm{C}$ will result in a $2.5 \mathrm{~cm}$ increase in sea level. Warming of this magnitude is consistent with observations at the coast (e.g., Enfield, 1981b).

It is striking that the amplitude of the second peak at the coast is correctly modeled while the first peak is considerably underpredicted. For any linear theory relying on the winds along the equator this result is an inevitable consequence of the fact that the collapse of the trades that produces the second peak is a much larger anomaly than the wind changes around the dateline that produce the first peak. (This difference is obvious in Fig. 3 and is a consistent feature of all El Niño wind analyses.) In this light the mystery is how the first observed peak manages to reach the same amplitude as the second one. The linear Kelvin wave theory cannot account for it without modification.

The discrepancy under discussion is highly nontrivial in the context of the search for a coupled airsea interactive theory for the entire El Niño cycle. One can construct scenarios where the initial pool of warm water that spreads westward from the South American coast alters the pattern of atmospheric heating enough to cause the mid-year collapse of the trades. The issue then becomes the cause of the initial warming. Our results raise the possibility that the wind changes at the dateline are too small to account for it. If the winds are not the cause then there would have to be some antecedent condition in the ocean that leads to the pile-up of mass at the eastern end of the Pacific. It is hard to see what it could be; certainly nothing obvious is suggested by the sea level data (e.g., Fig. 1). Wyrtki $(1975,1982)$ has emphasized the strengthening of the equatorial easterlies and concomitant buildup in sea level in the west that usually precedes an El Niño event. However, in a linear theory only the change in the wind is significant and the observed change is present in our forcing data set.

Another, and in our view more likely, possibility is that the initial peak is indeed caused by the wind changes at the dateline but that the resolutely linear theory used here understates the transfer from wind stress to sea level change. In particular, the linear theory disregards the fact that the thermocline slopes up to the east. Our calculation assumes a density profile characteristic of the vicinity of the dateline, the region of principal wind forcing. In this region the thermocline depth is characteristically $\mathrm{O}(175 \mathrm{~m})$ while at the eastern end of the equator the climatological thermocline is essentially at the surface.

The linear theory neglects the possibility that as the packet of Kelvin waves contributing to the first 
sea level peak approaches the coast it slows down and steepens in a manner loosely analogous to waves on a sloping beach. This steepening enhances the sea level response. The east-west tilt of the thermocline is greatly reduced by midsummer, when the collapse of the trades begins to generate the second peak. Hence there is no similar enhancement at that time. The possibility raised here is plausible, but requires a quantitative calculation. The steric effect due to sea surface warming is another mechanism left out of the theory that will have a greater influence on the first peak than on the second.

The two gravest baroclinic-modes are both important for the calculated sea level response and the two contribute about equally. As noted above, there is observational evidence which may be interpreted as showing that first baroclinic-mode Kelvin waves do cross the Pacific as required by theory (Knox and Halpern, 1982; Eriksen et al., 1983). However, although according to linear theory the second mode should have an equally strong expression in sea level, the same data fail to show it clearly. The second mode has considerable vertical shears in the upper ocean and it is possible that it cannot survive the strong mixing in the vicinity of the equatorial undercurrent. It would not be inconsistent with the data or our results to suppose that this is the case and that the process transfers energy into the first mode. Of course, such mode mixing goes beyond the linear theory.

Gill's (1982) data study implies that the mid-Pacific Ocean responds more or less like the first baroclinic mode, but the eastern Pacific response cannot be fit without using higher modes. Again, the data is not consistent with the structure for the ocean's response implied by linear theory.

In summary, the work reported here tends to support the theory that the coastal El Niño signal is a response to remote winds in the central and western Pacific and that the carrier is packets of equatorial Kelvin waves, but it also suggests that the linear theory will have to be modified to account for the initial warming.

Acknowledgments. This research was supported by NASA Grant NAGW-463 and NSF Grant OCE 8214771 .

\section{REFERENCES}

Bjerknes, J., 1966: A possible response of the atmosphere's Hadley cell to equatorial anomalies of ocean temperature. Tellus, 18, 820-829.

Busalacchi, A. J., and J. J. O'Brien, 1981: Interannual variability of the equatorial Pacific in the 1960's. J. Geophys. Res., 86, 10 901-10907.

_-.., Takeuchi and J. J. O'Brien, 1983: On the interannual winddriven response of the tropical Pacific Ocean. Hydrodynamics of the Equatorial Ocean, J. C. S. Hihoul, Ed., Elsevier, 155195.

Cane, M. A., 1979: The response of an equatorial ocean to simple wind stress patterns: II. Numerical results. J. Mar. Res., 37, 253-299.
- , and E. S. Sarachik, 1976: Forced baroclinic ocean motions. I: The linear equatorial unbounded case. J. Mar. Res., 34, 629-665.

- _ and —, 1977: Forced baroclinic ocean motions. II: The linear equatorial bounded case. $J$. Mar. Res., 35, 395-432.

_- , and _ 1979: Forced baroclinic ocean motions. III: The linear equatorial basin case. J. Mar. Res., 37, 366-398.

- and -1981 : The response of a linear baroclinic equatorial ocean to periodic forcing. J. Mar. Res., 39, 652-693.

_- and R. J. Patton, 1984: A numerical model for low-frequency equatorial dynamics. J. Phys. Oceanogr. 14, 1853-1863.

Enfield, D. B., 1981a: Annual and nonseasonal variability of monthly low-level wind fields over the southeastern tropical Pacific. Mon. Wea. Rev., 109, 2177-2190.

_ 1981 b: El Niño Pacific eastern boundary response to interannual forcing. Resource Management and Environmental Uncertainty, M. S. Glantz, and J. D. Thompson, Eds., Wiley \& Sons, 213-254.

Eriksen, C. C., M. B. Blumenthal, S. P. Hayes and P. Ripa, 1983; Wind-generated equatorial Kelvin waves observed across the Pacific Ocean. J. Phys. Oceanogr., 13, 1622-1640.

Gent, P. R., K. O'Neill and M. A. Cane, 1983: A model of the semiannual oscillation in the equatorial Indian Ocean. J. Phys. Oceanogr., 13, 2148-2160.

Gill, A. E., 1982: Changes in thermal structure of the equatorial Pacific during the 1972 El Niño as revealed by bathythermograph observations. J. Phys. Oceanogr., 12, 1373-1387.

_ 1983: An estimation of sea level and surface current anomalies during the $1972 \mathrm{El}$ Niño and consequent thermal effect. $J$ Phys. Oceanogr., 13, 586-606.

Godfrey, J. S., 1975: On ocean spindown. I: A linear experiment. J. Phys. Oceanogr., 5, 399-409.

Goldenberg, S. B., and J. J. O'Brien, 1981: Time and space variability of tropical Pacific wind stress. Mon. Wea. Rev., 109, 1190-1207.

Hurlburt, H. E., J. C. Kindle and J. J. O'Brien, 1976: A numerical simulation of the onset of El Niño. J. Phys. Oceanogr., 6, 621-631.

Knox, R. A., and D. Halpern, 1982: Long range Kelvin wave propagation of transport variations in Pacific ocean equatorial currents. J. Mar. Res., 40(Suppl), 329-339.

Leetma, A., 1983: The role of local heating in producing temperature variations in the offshore waters of the eastern tropical Pacific J. Phys. Oceanogr., 13, 467-473.

Lighthill, M. J., 1969: Dynamic response of the Indian Ocean to the onset of the southwest monsoon. Phil. Trans. Roy. Soc., A265, 45-92.

McCreary, J., 1976: Eastern tropical ocean response to changing wind systems: with application to El Niño. J. Phys. Oceanogr., 6, 632-645.

Meyers, G., 1982: Interannual variation in sea level near Truk Island-A bimodal seasonal cycle. J. Phys. Oceanogr., 12, 1161-1168.

Moore, D. W., and S. G. H. Philander, 1977: Modeling of the tropical ocean circulation. The Sea, Vol. 6 (Chap 8), E. D. Goldberg, I. N. Cave, J. J. O'Brien and J. H. Steek, Eds., Interscience, $1048 \mathrm{pp}$.

Philander, S. G. H., 1979: Nonlinear coastal and equatorial jets. $J$. Phys. Oceanogr., 9, 739-747.

Rasmusson, E. M., and T. H. Carpenter, 1982: Variations in tropical sea surface temperature and surface wind fields associated with the southern oscillation/El Niño. Mon. Wea. Rev., 110, 354-384.

Wyrtki, K., 1975: El Niño-The dynamic response of the equatorial Pacific ocean to atmospheric forcing. J. Phys. Oceanogr., 5, 572-584.

, 1977: Sea level during the 1972 El Niño. J. Phys. Oceanogr., 7, 779-787.

, 1979: The response of sea surface topography to the $1979 \mathrm{El}$ Niño. J. Phys. Oceanogr., 9, 1223-1231.

, 1982: The southern oscillation, ocean-atmosphere interaction and El Niño. J. Mar. Technol. Soc., 16, 3-10. 